\title{
Synthesis of Yttria Stabilized Zirconia by Sol-gel Precipitation Using PEG and PVA as Stabilizing Agent
}

\author{
Bramhe Sachin N.*, Young Pil Lee*, Tuan Dung Nguyen* and Kim Taik-Nam***† \\ *Department of Materials Science and Engineering, Graduate School of Pai Chai University, Daejeon 302-735, Korea \\ ***Department of Information and Electronic Materials Engineering, Pai Chai University, Daejeon 302-735, Korea
}

(Received June 27, 2013 : Received in revised form July 19, 2013 : Accepted August 8, 2013)

\begin{abstract}
There is increasing interest in zirconia as a dental material due to its aesthetics, as well as the exceptionally high fracture toughness and high strength that are on offer when it is alloyed with certain oxides like yttria. In recent years, many solution based chemical synthesis methods have been reported for synthesis of zirconia, of which the sol-gel method is considered to be best. Here, we synthesize zirconia by a sol gel assisted precipitation method using either PEG or PVA as a stabilizing agent. Zirconia sol is first synthesized using the hydrothermal method. We used $\mathrm{NaOH}$ as the precipitating agent in this method because it is easy to remove from the final solution. Zirconium and yttrium salts are used as precursors and PEG or PVA are used as stabilizers to separate the metal ions. The resulting amorphous zirconia powder is calcined at $900{ }^{\circ} \mathrm{C}$ for $2 \mathrm{~h}$ to get crystallized zirconia. XRD analysis confirmed the partially stabilized zirconia synthesis in all the synthesized powders. SEM was taken to check the morphology of the powder synthesized using either PEG or PVA as a stabilizing agent and finally the transparency was calculated. The results confirmed that the powder synthesized with $10 \%$ PVA as the stabilizing agent had highest percentage of transparency among all the synthesized powder.
\end{abstract}

Key words zirconia, crystal structure, PEG, PVA, sol-gel, precipitation.

\section{Introduction}

Zirconium is the transition element which reacts with oxygen to form zirconium dioxide(Zirconia) at $400{ }^{\circ} \mathrm{C} .{ }^{1)}$ Zirconia $\left(\mathrm{ZrO}_{2}\right)$ has garnered wide scale attention due to its application in variety of fields. ${ }^{2,3)}$ This is due to the excellent electrical, optical, thermal and mechanical properties. ${ }^{4-6)}$ It has high elastic modulus, hardness, low friction coefficient, high boiling point, and is stable in chemically aggressive environment and resist detrition. ${ }^{1,7,8)}$ All these properties have helped $\mathrm{ZrO}_{2}$ gather attention as a dental material. Zirconium dioxide has three stable structures namely monoclinic, tetragonal and $\mathrm{cubic}^{4)}$ and it can also form orthorhombic structure. ${ }^{6}$ The monoclinic structure is stable at temperature below $1170{ }^{\circ} \mathrm{C}$, tetragonal structure is stable at temperature between $1170^{\circ} \mathrm{C}$ $2370{ }^{\circ} \mathrm{C}$ and cubic structure is stable at temperature above $\left.2370{ }^{\circ} \mathrm{C} .{ }^{1,5}\right)$ Excellent mechanical properties are shown by tetragonal phased $\mathrm{ZrO}_{2}{ }^{\left.{ }^{9}\right)}$ When heated, $\mathrm{ZrO}_{2}$ can be transformed into tetragonal phase but when it is brought back to room temperature, it again transforms into monoclinic form with small percentage of volume change. In a tetragonally stable zirconia block, for crack propagation energy has to be dissipated to transform $\mathrm{ZrO}_{2}$ from tetragonal to monoclinic form and to overcome the compressive stress caused due to volume change. This leads to improved mechanical strength of the material. Because of this, $\mathrm{ZrO}_{2}$ is being studied as a dental material and there is a need of doping agents which can help in stabilization of tetragonal or cubic form at room temperature. Some examples of doping material that can be used are $\mathrm{Y}_{2} \mathrm{O}_{3}, \mathrm{CaO}, \mathrm{MgO}, \mathrm{Ce}_{2} \mathrm{O}, \mathrm{Gd}_{2} \mathrm{O}_{3}, \mathrm{Sc}_{2} \mathrm{O}_{3}$ etc. ${ }^{1,8,10,11)}$

Studies have shown that $8 \mathrm{~mol} \%$ yttria helps in stabilization of $\mathrm{ZrO}_{2}$ in cubic form, whereas $3 \mathrm{~mol} \%$ yttria helps in stabilization of $\mathrm{ZrO}_{2}$ in tetragonal form. ${ }^{2,10)}$ There are a number of methods by which yttria stabilized $\mathrm{ZrO}_{2}$ can be synthesized like sol-gel, hydrothermal synthesis, precipitation, thermal decomposition, etc. ${ }^{1,2,12)}$ Sol-gel method is considered to be the best method for this syn-

†Corresponding author

E-Mail : tnkim@pcu.ac.kr (T.-N. Kim, Pai Chai Univ.)

(C) Materials Research Society of Korea, All rights reserved.

This is an Open-Access article distributed under the terms of the Creative Commons Attribution Non-Commercial License (http://creativecommons.org/licenses/by-nc/3.0) which permits unrestricted non-commercial use, distribution, and reproduction in any medium, provided the original work is properly cited. 
thesis. Sol-gel method helps in controlling the porosity ${ }^{2)}$ thus also helping in controlling the translucency. In the present study, yttria stabilized $\mathrm{ZrO}_{2}$ is synthesized by combining sol-gel method with precipitation method. We have used polyethylene glycol and polyvinyl alcohol in the process as a stabilizing agent. The long chains of polymer make sure that the metal ions are homogenously distributed and do not aggregate or precipitate in the solution. ${ }^{13)}$ The precursor used is inorganic zirconium salt which is relatively inexpensive and insensitive to moisture. ${ }^{7}$

\section{Experimental}

$3 \mathrm{~mol} \%$ yttria stabilized $\mathrm{ZrO}_{2}$ was synthesized by using zirconium chloride octahydrate(Junsei, Japan) and yttrium chloride(Aldrich, USA) as precursors. $\mathrm{ZrO}_{2}$ was synthesized with a combination of sol-gel and precipitation methods. The process is depicted in Fig. 1. First $12.89 \mathrm{~g}$ $\mathrm{ZrOCl}_{2} .8 \mathrm{H}_{2} \mathrm{O}$ and $0.36 \mathrm{~g} \mathrm{YCl} 3.6 \mathrm{H} 2 \mathrm{O}$ are added to $40 \mathrm{ml}$ D.I. water and autoclaved at $180{ }^{\circ} \mathrm{C}$ for $24 \mathrm{~h}$. After bringing the solution to room temperature, supernatant was discarded and mixture solution of $1 \mathrm{~N} \mathrm{NaOH}$ and stabilizing agent was added to the precipitate along with mild stirring and heating at $70{ }^{\circ} \mathrm{C}$. As shown in the figure, the experiments were performed either by using PEG or PVA as stabilizing agent. The residual $\mathrm{Cl}^{-}$ions present in the precipitate from the precursors have to be removed from the final product. To this end the precipitate was washed four times with water and twice with absolute ethanol. The resulting precipitation was dried in the oven at $80{ }^{\circ} \mathrm{C}$ overnight and finally calcined at $900{ }^{\circ} \mathrm{C}$ for $2 \mathrm{~h}$ in a box furnace.

The $\mathrm{ZrO}_{2}$ powder thus synthesized was characterized using X-ray diffraction, Scanning electron microscopy and finally transparency of each sample was determined. Crystal structure was determined by X-ray Diffraction (XRD, CuK $\alpha 1$ radiation $(\lambda=0.15406 \mathrm{~nm}), \mathrm{D} 1 \mathrm{w}$, Shimadzu, Japan) over the range of $20^{\circ}$ to $80^{\circ}$ at a scan rate of $2^{\circ} \%$ min. SEM images were taken using SEM Hitachi S-4800 model. For transparency evaluation samples were sent to Dentium Co. Ltd., South Korea.

\section{Result and Discussion}

Zirconia, at room temperature, can be in purely monoclinic form, can be synthesized in purely tetragonal form or it can have monoclinic and tetragonal crystal structure both. ${ }^{1,5,11)} \mathrm{ZrO}_{2}$ is mechanically stronger and has illustrious application when its crystal structure is a combination of tetragonal and monoclinic. ${ }^{9)}$ This type of material is called as partially stabilized zirconia(PSZ). The tetragonal to monoclinic transformation imparts mechanical strength to the material. During crack propagation in a PSZ material,

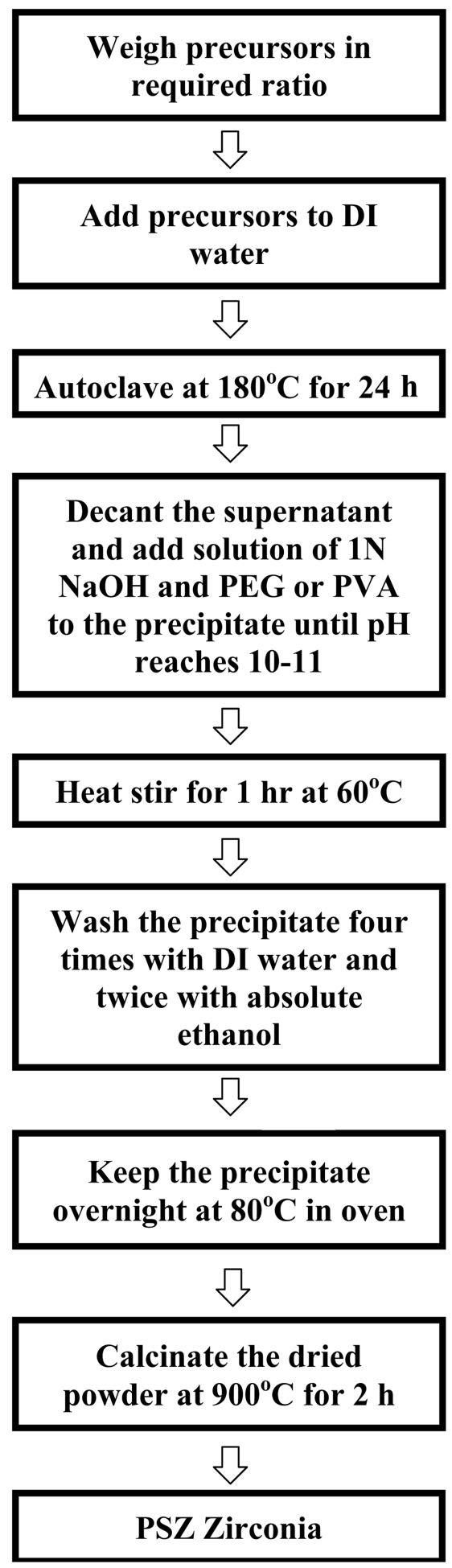

Fig. 1. Schematic representation of steps followed for synthesis of zirconia.

energy is required to transform tetragonal crystal structure into monoclinic crystal structure. This transformation is accompanied with a volume increase by approximately $4 \%$. Due to this volume increase, a compressive stress is developed near the crack. During crack propagation, 
energy is dissipated in transformation from tetragonal to monoclinic as well as to overcome the compressive stress. Thus it increases the strength of the material.

$\mathrm{ZrO}_{2}$ was synthesized using co-precipitation and sol-gel method. When the precursors are dissolved in D.I. water, the $\mathrm{pH}$ of the solution becomes highly acidic due to the $\mathrm{Cl}^{-}$ions. When $\mathrm{NaOH}$ is added the $\mathrm{pH}$ slowly increases and in the process $\mathrm{ZrO}_{2}$, which is insoluble in water, precipitates. We think that during this process $\mathrm{NaCl}$ is also formed. This $\mathrm{NaCl}$ can be withdrawn by washing with water. When we heat the solution again, DI water starts to evaporate. Due to this the viscosity of the solution increases and it turns into gel. This gel is then dried overnight and finally calcined at $900{ }^{\circ} \mathrm{C}$.

The molar concentration of $\mathrm{ZrO}_{2}$ used earlier by other groups is generally very $\operatorname{low}(<0.5 \mathrm{M})$. Here we have tried the synthesis with concentration of 1 molar. We have synthesized $\mathrm{ZrO}_{2}$ in two groups. In one group we have used PEG as a stabilizing agent for the synthesized

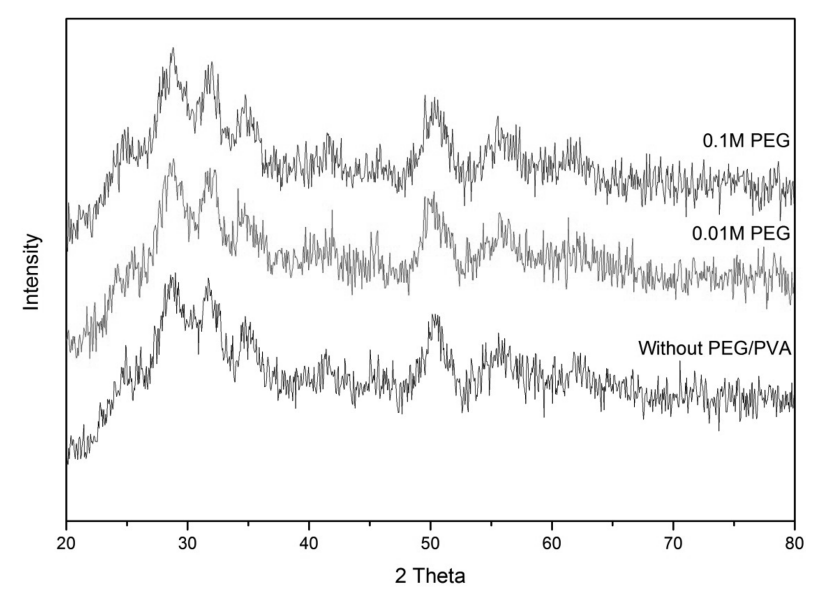

Fig. 2. XRD pattern of control sample without PEG or PVA and that of sample from group 1 with $0.01 \mathrm{M}$ PEG and 0.1 M PEG, all showing amorphous nature before calcination.

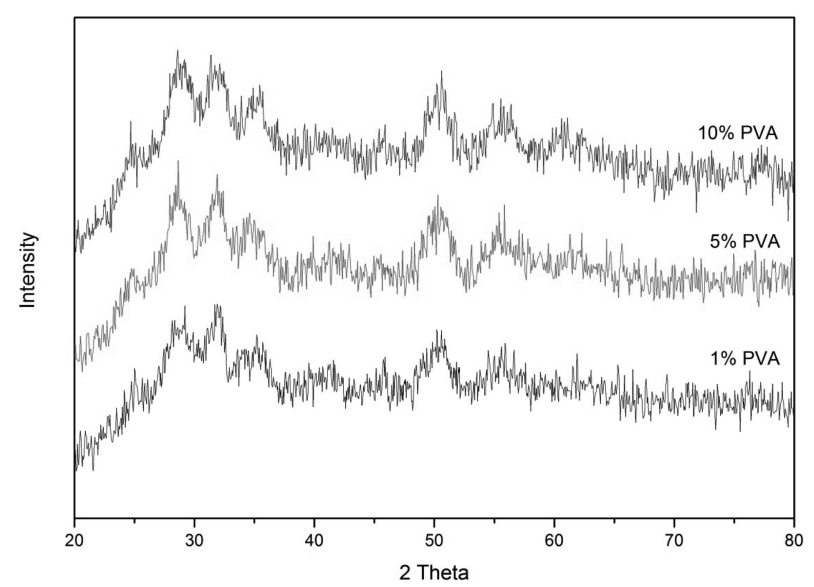

Fig. 3. XRD pattern of sample from group 2 with $1 \%, 5 \%$ and 10 $\%$ PVA, all showing amorphous nature before calcination.
$\mathrm{ZrO}_{2}$ and in the other group we have used PVA as a stabilizing agent. We have studied the difference in transparency of both groups.

\subsection{XRD measurement}

$X$-ray diffraction pattern was studied to analyse the crystal structure of the synthesized $\mathrm{ZrO}_{2}$ powder. XRD analysis of both groups shows that the $\mathrm{ZrO}_{2}$ is in amorphous form before calcination as can be seen in Fig. 2 and 3. And after calcination at $900{ }^{\circ} \mathrm{C}$ for $2 \mathrm{~h}, \mathrm{ZrO}_{2}$ has a combination of both monoclinic and tetragonal structure. This is true for all the synthesized samples as can be seen in Fig. 4 and 5. Thus XRD analysis confirms the synthesis of partially stabilized $\mathrm{ZrO}_{2}$ in all the samples. The residual chloride ions present after purification step pose a problem in obtaining pure zirconia powder. These ions are difficult to remove completely which results in some excess peaks in the XRD pattern. These chloride

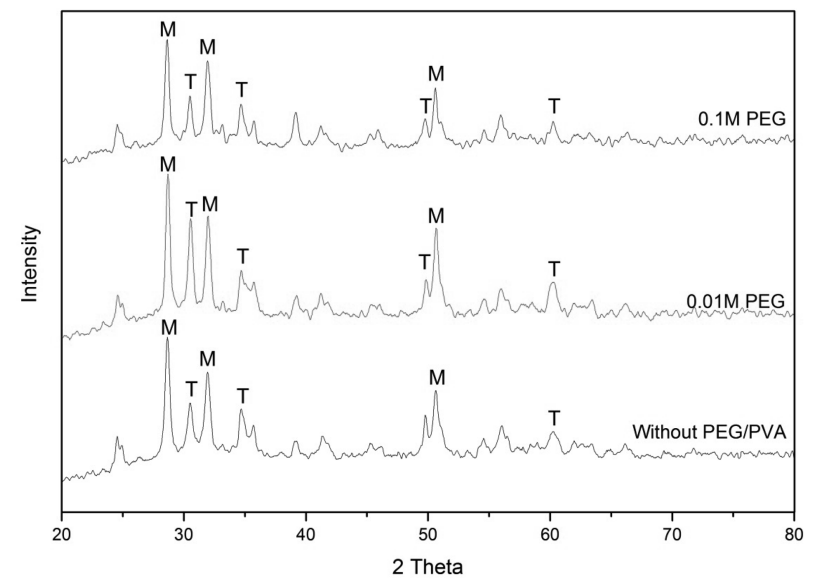

Fig. 4. XRD pattern of control sample without PEG or PVA and that of samples from group 1 with $0.01 \mathrm{M}$ PEG and 0.1 M PEG, all showing monoclinic(M) and tetragonal(T) structure after calcination.

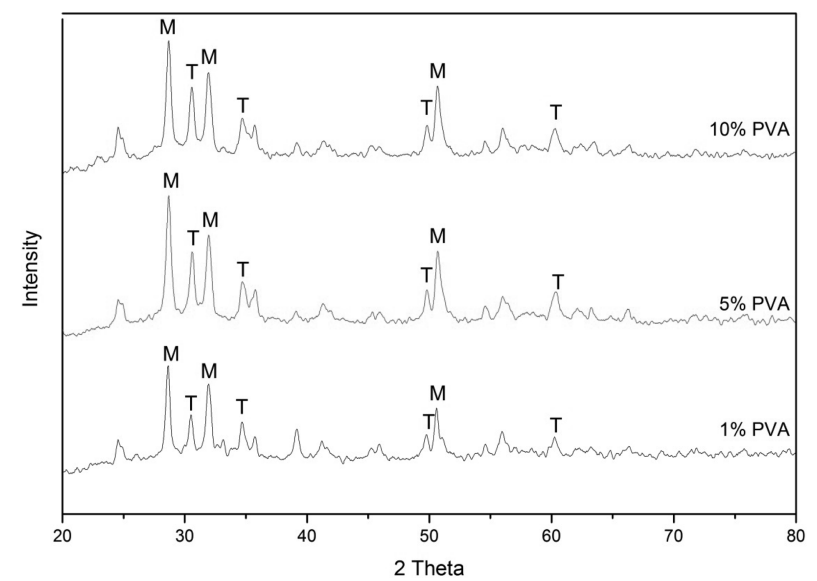

Fig. 5. XRD pattern of samples from group 2 with $1 \%, 5 \%$ and $10 \%$ PVA, all showing monoclinic(M) and tetragonal(T) structure after calcination. 


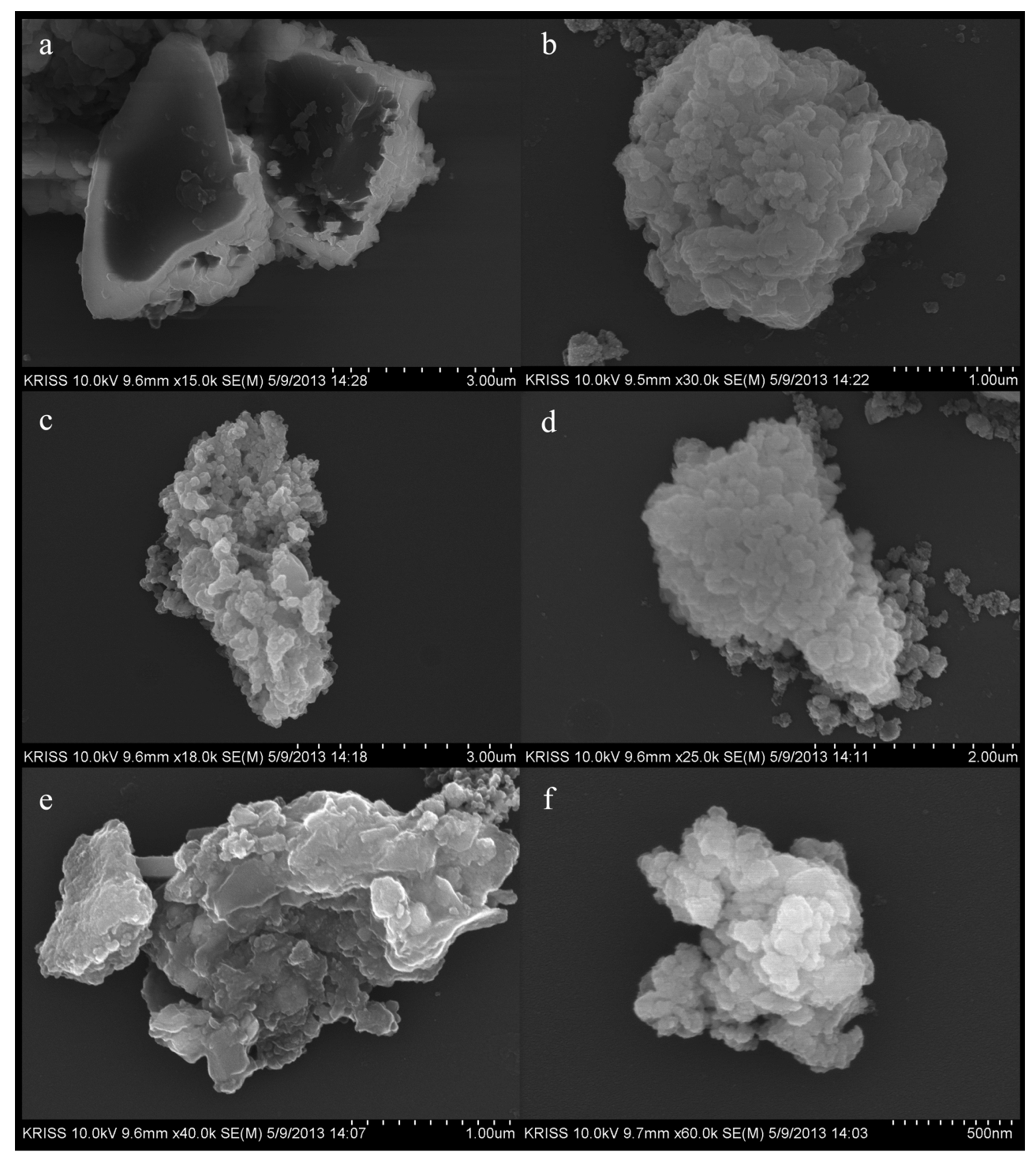

Fig. 6. SEM image of zirconia control sample (a), zirconia synthesized with $0.01 \mathrm{M}$ PEG (b), $0.1 \mathrm{M}$ PEG (c), 1 \% PVA (d), $5 \%$ PVA (e) and $10 \%$ PVA (f) before calcination. All structures are amorphous in nature.

ions are also believed to cause aggregation and thus increase the particle size. More extensive purification steps are needed to get completely rid of the said $\mathrm{Cl}^{-}$ions.

\subsection{Scanning Electron Microscopy}

The sample morphology was studied by using SEM. Morphology as seen for samples before calcination is shown in Fig. 6 and after calcination is shown in Fig. 7. All the samples before calcination are amorphous in nature hence shows no effect of stabilizers, whereas the crystallized $\mathrm{ZrO}_{2}$ obtained after calcination show different morphology at different experimental conditions. The control sample containing neither PEG nor PVA as a stabilizing agent shows morphology ranging from ablated sphere to rods. This might be because no stabilizing agent is present, so the structure is free to grow in any dimension. Sample with 0.01 M PEG also shows ablated spheres as well as rods. Also some plate like structure can be seen which shows that $\mathrm{ZrO}_{2}$ is not stabilized and aggregation takes place. Sample with $0.1 \mathrm{M}$ Peg shows ablated spherical morphology with size of $\sim 90 \mathrm{~nm}$. Rod shaped morphology was seen in sample with $1 \%$ and $10 \%$ PVA and only spherical morphology with size $\sim 70 \mathrm{~nm}$ was seen in sample stabilized with $5 \%$ PVA. We believe, the structure of $\mathrm{ZrO}_{2}$ is stabilizing agent concentration dependent. More studies needs to be done to ascertain this phenomenon and this may even lead to different avenues for application of $\mathrm{ZrO}_{2}$.

\subsection{Transparency}

Transparency is the property of being transparent or allowing light to pass. If we have small sized adequately 


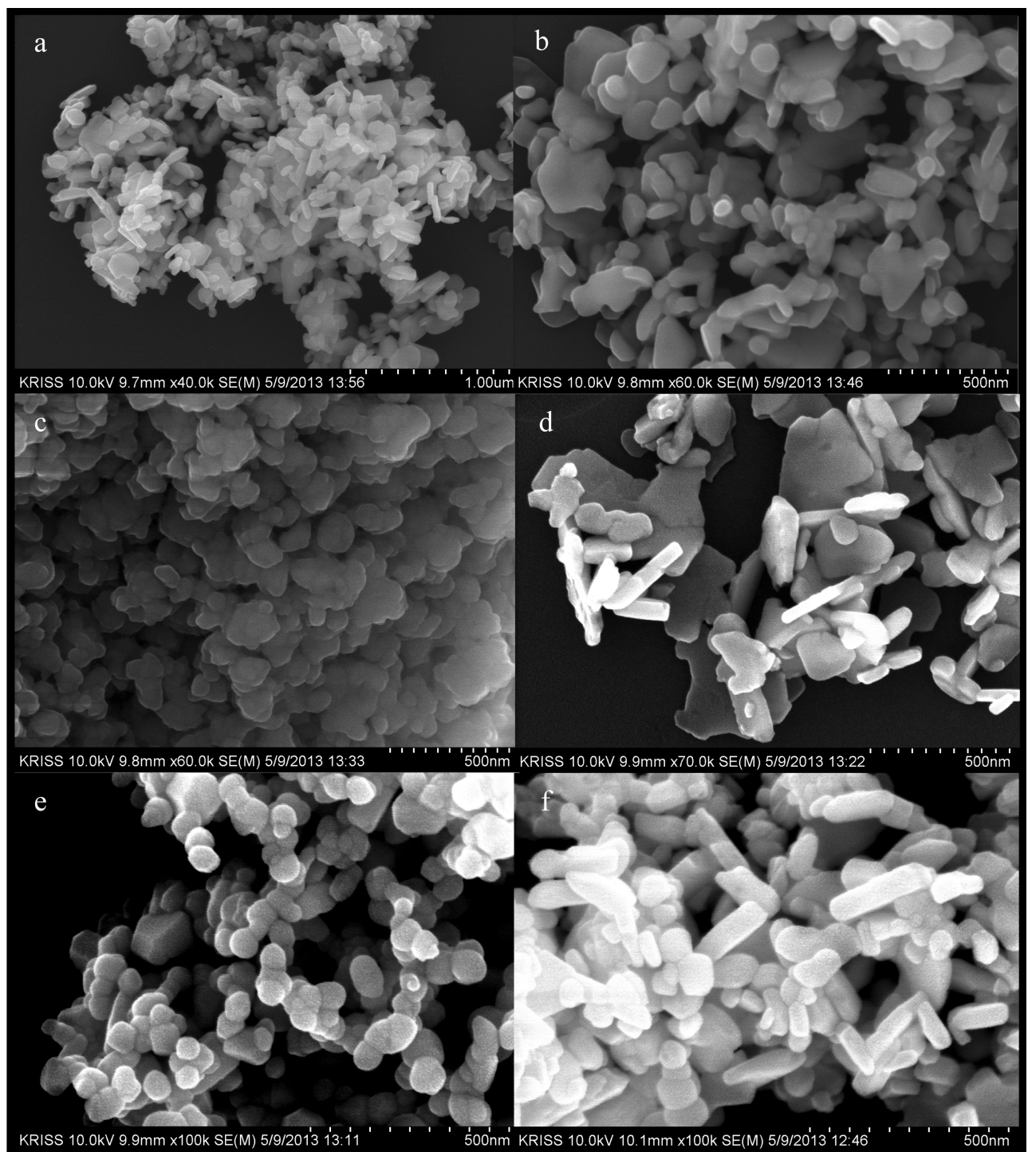

Fig. 7. SEM image of zirconia control sample (a), zirconia synthesized with 0.01 M PEG (b), 0.1 M PEG (c), $1 \%$ PVA (d), $5 \%$ PVA (e) and $10 \%$ PVA (f) after calcination.

Table 1. Table showing thickness and diameter of the disks made to evaluate the transparency of zirconia along with the transparency observed.

\begin{tabular}{ccccc}
\hline Sample & Composition & Thickness(mm) & Diameter(mm) & Transparency(\%) \\
\hline Control & $1 \mathrm{M} \mathrm{NaOH}+$ No PEG or PVA & $1.18 \pm 1.20$ & $17.82 \pm 17.81$ & $6.10 \pm 6.03$ \\
Group 1 Sample 1 & $1 \mathrm{M} \mathrm{NaOH}+0.01 \mathrm{M}$ PEG & $1.42 \pm 1.41$ & $18.87 \pm 18.86$ & $1.73 \pm 1.96$ \\
Group 1 Sample 2 & $1 \mathrm{M} \mathrm{NaOH}+0.1 \mathrm{M} \mathrm{PEG}$ & $1.37 \pm 1.37$ & $16.06 \pm 16.08$ & $8.90 \pm 8.89$ \\
Group 2 Sample 1 & $1 \mathrm{M} \mathrm{NaOH}+1 \%$ PVA & $1.28 \pm 1.21$ & $18.52 \pm 18.56$ & $3.88 \pm 4.10$ \\
Group 2 Sample 2 & $1 \mathrm{M} \mathrm{NaOH}+5 \%$ PVA & $1.15 \pm 0.98$ & $18.95 \pm 18.90$ & $7.12 \pm 7.15$ \\
Group 2 Sample 3 & $1 \mathrm{M} \mathrm{NaOH}+10 \%$ PVA & $1.21 \pm 1.20$ & $17.95 \pm 18.03$ & $13.49 \pm 13.45$ \\
\hline
\end{tabular}

dispersed particles it would allow more light to pass through the block as opposed to aggregates which will block the incident light. Hence two conditions seems to be necessary for achieving greater transparency, first that the grain size should be low and second that the particles should be adequately stabilized so as to oppose formation of aggregates. The transparency of each synthesized sample was as shown in Table 1 . It can be seen that the sample synthesized using $10 \%$ PVA as stabilizing agent has highest transparency(13.49\%) among the synthesized 
samples. This might be because the higher concentration of PVA helps in better stearic stabilization of synthesized $\mathrm{ZrO}_{2}$. We believe that PEG is not able to stabilize $\mathrm{ZrO}_{2}$ as good as PVA, hence the lower transparency. Aggregates of $\mathrm{ZrO}_{2}$ blocks the light and hence allows less light to penetrate thus decreasing the transparency. A properly stabilized $\mathrm{ZrO}_{2}$ sample thus would increase the transparency as is observed in our results. Addition of small percentage of photocatalyst $\mathrm{TiO}_{2}$ could increase the transparency of $\mathrm{ZrO}_{2}$. $\mathrm{TiO}_{2}$ has high diffraction index, shows strong light scattering and incident-light reflection capability. Hence addition of $\mathrm{TiO}_{2}$ to the $10 \%$ PVA sample could further increase the transparency.

\section{Conclusion}

We have synthesized $\mathrm{ZrO}_{2}$ with higher concentration of precursor material which was never tried before. The use of this higher concentration would save resources while synthesizing $\mathrm{ZrO}_{2}$ at larger amount. We have shown that the synthesis method applied, results in synthesis of partially stabilized $\mathrm{ZrO}_{2}$. We then compared the transparency results of two groups where we have used PEG and PVA as stabilizing agent and we found that $10 \%$ PVA gives better transparency among the other synthesized $\mathrm{ZrO}_{2}$. There is a need to find other good stabilizing agents which would provide better stability and thus increase the transparency. Also, there is a need for extensive purification steps to obtain purified Zirconia without any excess ions. Further analysis needs to be done to ascertain the use of this synthesized zirconia as a dental material.

\section{References}

1. Antonio Díaz-Parralejo, Antonio Marias-Garcia, José Sánchez-González, M. Ángeles Díaz-Díez and Eduardo M. Cuerda-Correa, J. Non-Crystalline Solids, 357, 1090 (2011).
2. Zijun Zhang, Jiurong Liu, Fenglong Wang, Jing Kong and Xinzhen Wang, Ceram. Int., 37, 2549 (2011).

3. J. A. Wang, M. A. Valenzuela, J. Salmones, A. Vázquez, A. Garcia-Ruiz and X. Bokhimi, Catalysis Today, 68, 21 (2001).

4. Felora Heshmatpour and Reza Babadi Aghakhanpour, Powder Technol., 205, 193 (2011).

5. Javier Rivera De la Rosa, Aracely Hernandez, Fernando Rojas, Juan José Ledezma, Colloids and Surfaces A: Physicochemical and Engineering Aspects, 315, 147 (2008).

6. Zeming Qi, Chaoshu Shi, Yaguang Wei, Zheng Wang, Tao Liu, Tiandou Hu, Zongyan Zhao and Fuli Li, J. Phys.: Condens. Matter, 13, 11503 (2001).

7. Weimin Liu, Yunxia Chen, Chengfeng Ye, Pingyu Zhang Ceram. Int.1 28, 349 (2002)

8. X. Bokhimi, A. Morales, A. Garcia-Ruiz, T. D. Xiao, H. Chen and P. R. Strutt, J. Solid State Chemistry, 142, 409 (1999).

9. Oleg Vasylvik and Yoshio Sakka, J. Am. Ceram. Soc., 84, 2489 (2001).

10. S. Salehi and M. H. Fathi, Ceram. Int., 36, 1659 (2010).

11. R. Ramamoorthy, D. Sundararaman and S. Ramasamy, Solid State Ionics, 123, 271 (1999).

12. Qibing Chang, Jian-er Zhou, Yongqing Wang and Guangyao Meng, Advanced Powder Technology, 21, 425 (2010).

13. Sang Jin Lee, Hyun-Kwuon Lee and Kyeong-Sik Cho J. Ceram. Processing Research, 6, 85 (2005). 